\title{
Experimental RF-Signal Based Wireless Energy Transmission
}

\author{
Janne Janhunen, Konstantin Mikhaylov, Juha Petäjäjärvi \\ Centre for Wireless Communications, University of Oulu, Finland \\ Solmu Technologies Ltd. \\ janne.janhunen@oulu.fi
}

\begin{abstract}
The distributed measurement and control systems employing wireless connectivity are commonly seen as the key enablers for many novel industry, retail and consumer applications. Even though, the fast spread of large wireless sensor networks today is obstructed by the absence of solutions, which are at the same time dependable, and can minimize both capital and operational expenditures. In this respect the devices, which are powered with the energy collected from their environment, look very attractive. Among all of them, the systems collecting the energy from a designated wireless (i.e., radio frequency (RF)) channel can often enjoy a more stable energy income, which makes them more dependable than their counterparts. Due to this reason, in the current paper we focus on and discuss the key techniques and design aspects enabling development of a real-life RF-powered wireless sensor device. Namely, we address the problems of designing an antenna and the energy harvesting circuit with feasible efficiency. In addition, we detail particular aspects related to the design of a sensor node and optimizing its computing and communication. The developed device is tested in harsh environment as a part of a fast rotating mechanical structure, showing the feasibility of the proposed solutions. The technology discussed in the paper is an important part of the upcoming 5G and IoT development and deployment.
\end{abstract}

\section{INTRODUCTION}

Wireless Sensors Networks (WSNs) are a critical component and a fundamental enabler of the evolving $5 \mathrm{G}$ and IoT applications. These applications vary from military to health and wellbeing monitoring, from environment sensing to vehicle tracking [1] and include many other versatile home, industry and consumer applications. Some of these use cases involve deployment of hundreds or even thousands of miniature embedded devices, called nodes. Each node usually includes a transceiver, a microcontroller, the set of peripherals (typically sensors and actuators) and an energy source, typically represented by primary or secondary battery. Nonetheless, an ultra low power signal processing and wireless communications provide possibilities to apply an energy harvester as part of the design enabling a battery free sensor application.

The nodes in the network do not necessary rely on a pre-existing architecture but depend on a self-forming infrastructure and connect between themselves by using configurations based on the application as well as on the environment [2]. The capability of communicating over wireless channels and without batteries allows deployment in harsh environments, for instance, inside an engine where vibrations and moving objects would abstain from placing the sensors and using heavy and volatile batteries. On the other hand, an extremely large numbered sensor network operating without batteries reduces the maintenance costs. Therefore, environments and applications in which using sensors was impossible or too expensive have now become reality.

In order to exploit energy transmission or harvesting, the energy consumption of a node should be balanced with the available energy. For wireless communication, IEEE 802.15.4 standard [3] offers a low energy consumption, low complexity circuitry and low-cost radio technology. IEEE 802.15.4a features an additional PHY layer and provides location capability as well as high ranging precision. In this research, we apply Bluetooth Low Energy (BLE) for wireless communication in rotating enviroment. We have confirmed in previous studies [4], that the IEEE 802.15.4 based Zigbee and UWB radios can operate in challenging rotating enviroment, nonetheless the consumption of these radios (especially the latter) still introduces substantial challenge when attempting to power them with energy harvesting. Due to this reason, in the current study we have used the BLE, which due to higher over-the-air rate and the absence of listen before talk requires few time less energy than Zigbee.

Wireless energy transmission has been an interest of the research community in recent years. A comprehensive survey of wireless energy transmission has been presented in [5] and in references therein. The survey summarizes the critical sources of losses such as impedance matching, component parasitics and harmonic generation. The research presents various energy-harvesting circuit topologies and state-of-the-art Schottky diode based circuit designs summarizing implementation efficiencies. The efficiencys are reported between 1.272.8 percent in $869-2450 \mathrm{MHz}$ frequency range. Another research [6] focuses on optimization and implementation aspects of the low power energy harvesters. The authors model, design and implement an energy harvester including antennas. The reported efficiency for the energy harvesting circuit is 20 to 54 percent for $-35-10 \mathrm{dBm}$ strong RF signal on input. In addition, the results show that the transmitted signal has to be strong enough and the antennas optimized to receive sufficient DC power.

The key contributions of this paper are as follows. First, we discuss the possibilities for energy harvesting and advocate the use of energy transfer in the RF channels. Importantly, we show that under the frequency regulations of today under some preconditions almost continuing transfer of energy can be enabled. Second, we discuss the major design aspects of the three main components of a wireless energy transfer powered sensor node, namely its energy receiving antenna, rectifying circuit and the sensor node itself. All the discussions are backed up by the practical implementation and evaluation activities. Finally, we show the feasibility of our approaches by building a real-life demo application, which confirms the potential possibility of developing an industrial sensory application powered solely with the energy transferred over an RF channel.

The rest of the paper is organized as follows. Section II discusses the various aspects related to energy harvesting in general and wireless energy transmission in particular. Importantly, the section gives insight in the radio regulations in the context of wireless energy transfer in RF. Section III summarizes the most critical aspects for designing an antenna to be used for energy transmission. Section IV discusses the design of an energy harvesting circuit and the ways for improving its performance. Section V details some of the critical aspects related to the design of a sensor node to be powered with energy harvesting. Our demo experimental setup is detailed in Section VI which is followed by Section VII presenting the conclusions. 


\section{ENERGY HARVESTING AND TRANSMISSION}

\section{A. Options}

Depending on the application, several possibilities for harvesting the energy from environment (i.e., collect the energy from ambient sources) or specifically transferring it to a node (i.e., designated emission of a signal to provide energy for one or multiple nodes) do exist.

Even though the energy harvesting with photovoltaic panels is already a mature technology, it continues to evolve. As of today the maximum efficiency of a photovoltaic cell, as shown in [7], does not exceed $46 \%$. However, the usability of photovoltaic energy harvesting for many indoor applications is limited even more since due to the narrow bandwidth of the artificial indoor lightning spectrum and less than 20 percent of outdoors efficiency can be achieved. Albeit the ideas of intentional transfer of energy with light (e.g., using lasers or as a part of visual light communication (VLC) systems) have been expressed, the authors are not aware of any functional implementation of such a system.

Piezoelectric elements enable harvesting energy from a variety of physical impacts and processes, like hits or vibration. An optimized piezo harvester can collect energy up to tens or hundreds of millWatts within one $\mathrm{cm}^{3}$ volume. The performance of the piezoelectric harvesters is strongly affected by acceleration, structural design, applied force, vibration frequency, impedance matching, mass, and surrounding environment and materials. A recent review study on piezoelectric progress is presented in [8]. Interestingly, the piezoelectric devices benefit from the scaling laws as they can keep up the high performance even with miniaturized size while being less efficient in macro-sized structures compared to electromagnetic devices. As this goes with the light, the piezoelectric elements are typically employed for the opportunistic energy harvesting and the authors are not aware of any single solution employing dedicated energy transfers with piezoelectric harvesters.

Electromagnetic induction [9] can be applied for both energy harvesting (e.g., created by the current flowing in a cable) and transmission within a short range. The technique scales decently, but the physical size of the coils and the direction of magnetic fields and coils tends to obstruct the use of this technique for particular applications. The technique is promising in applications such as wireless mobile device chargers or electric car chargers, where the physical size of the coils are not a major limiting factor and where the mutual position of the devices are not assumed to change drastically. Another substantial problem which somewhat limits the use of inductive solutions especially in industrial applications is the negative effect the presence of conductive materials between the coils may have on the energy transfer performance [10].

The radio frequencies (RF) around us carry a lot of energy, thus providing an interesting opportunity for powering a low-consuming device. As this comes with induction, one can either harvest the "free" energy from radio frequencies (radio, TV, cellular or WiFi signals, radiation of radars, etc.) or may establish a designated link purposed to deliver the energy to a device. The first solution works well for opportunistic ultra low power applications which can harvest and store energy before acting, whereas the latter technique enables ultra low power real-time operation. One of the major limitations for the latter are the restrictions imposed by the radio frequency regulations, which are briefly discussed below.

Table I summarizes the reported energy volumes for different techniques [11]. In RF energy transmission radio regulations are taken into account. In this paper, we have limited our study to the techniques for energy harvesting from the radio frequencies and particularly to the short range energy transmission.
TABLE I. HARVESTED ENERGY WITH DIFFERENT TECHNIQUES

\begin{tabular}{lr} 
Energy source & Harvested power \\
\hline Light (outdoor/indoor) & $10 / 0.01 \mathrm{~mW} / \mathrm{cm}^{2}$ \\
Electromagnetic field & $0.1-4500 \mathrm{~mW} / \mathrm{cm}^{3}$ \\
Temperature difference & $1-10 \mathrm{~mW} / \mathrm{cm}^{2}$ \\
Vibration/motion & $0.2-231 \mathrm{~mW} / \mathrm{cm}^{3}$ \\
RF energy transmission & $0.1 \mathrm{~mW}-100 \mathrm{~mW}$ \\
\hline
\end{tabular}

\section{B. Radio Regulations}

The use of radio frequencies in Finland are governed by the Finnish Communications Regulatory Authority (Ficora), which acts in concert with European Commission and whose documents (e.g., [12]) are based on the ERC recommendations. Note, that as of today no specific regulatory basis for far field RF-based energy transfer do exist. Due to this reason in the current section we focus specifically on the regulation governing the communication for nonspecific short range devices operating in ISM (industrial, scientific and medical) radio bands, which can be employed for energy transfers. Table II summarizes the effective radiated power (ERP), equivalent isotropically radiated power (EIRP) and duty cycle restrictions in the selected Region 1 bands. Note that the duty cycle is defined as the ratio, expressed as percentage, of the maximum transmitter on time, relative to one hour period.

TABLE II. SUB-BANDS FOR NON-SPECIFIC SHORT RANGE DEVICES

\begin{tabular}{lrr} 
Sub-band $[\mathrm{MHz}]$ & ERP $[\mathrm{mW} / \mathrm{dBm}]$ & Duty cycle $(\%)$ \\
\hline $433.050-434.790$ & $\leq 25 / 14$ & $\leq 10$ \\
$433.050-434.790$ & $\leq 1 / 0(-13 \mathrm{dBm} / 10 \mathrm{kHz})$ & No restriction \\
$433.040-434.790$ & $\leq 10 / 10$ & No restriction \\
468.200 & $\leq 500 / 27$ & No restriction \\
$863.000-870.000$ & $\leq 25 / 14$ & $\leq 0.1$ \\
$868.000-868.600$ & $\leq 25 / 14$ & $\leq 1$ \\
$868.700-869.200$ & $\leq 25 / 14$ & $\leq 0.1$ \\
$869.400-869.650$ & $\leq 500 / 27$ & $\leq 10$ \\
$869.700-870.000$ & $\leq 5 / 7$ & - \\
$869.700-870.000$ & $\leq 25 / 14$ & $\leq 1$ \\
$870.700-873.000$ & $\leq 25 / 14$ & $\leq 1$ \\
$2400.000-2483.500$ & $\leq 10 / 10$ (EIRP) & No restriction \\
\hline
\end{tabular}

In Europe, spectrum access was originally regulated only on the duty cycle basis (as shown in Table II) but later the possibility to employ an appropriate access protocol and egress the restrictions was introduced. In practice, an appropriate access protocol means Listen Before Talk (LBT) protocol with preferred option of Adaptive Frequency Agility (AFA) which enables a dynamic channel change within available frequencies. If such a protocol is used, a device is entitled to transmit non-stop for maximum of $1 \mathrm{~s}$ after confirming that a channel is free using LBT. At the same time a device is restricted to operate for only $100 \mathrm{~s}$ within one hour for any $200 \mathrm{kHz}$ frequency band [13]. As one can see, this potentially enables almost non-stop transmission of the energy e.g., the $10 \mathrm{MHz}$ bandwidth of the 868 MHz band enables up to $99,5 \%$ (note 5 ms minimum time for channel check) on-air time. Even though, as one can see from Table II the highest transmit power is allowed only in one sufficiently narrow band. Another challenge introduced by this approach is the need of having a sufficiently broadband antenna (over 7.2 MHz) to collect the energy within all the bands used for AFA.

\section{ANTENNA DESIGN FOR ENERGY TRANSMISSION}

The design of transceiver antennas is of extreme importance for enabling highly efficient wireless energy transfer. The well designed receiver antenna should maximize the amount of radio signals energy it collects from the air and provides further to the AC-DC converter 
circuitry. This calls for maximizing the aperture of such an antenna. Nonetheless the requirements of the real-life applications often limit the antennas dimensions. At the very same time, the major purpose of the transmitters antenna is to provide the maximum amount of energy to one or multiple receivers. If there is only one receiver or if the receivers are tightly grouped, a directive antenna can be employed. In the ultimate case, when the width of the beam at the receive antenna is below or close to its aperture the major part of the energy can be effectively recovered and the efficiency of the system becomes higher [14]. Note that given that the position of the receivers or transmitters are known, the multiple-input multiple-output (MIMO) or multipleinput single-output (MISO) techniques can be employed for wireless energy transfer. On the other hand, if the positions of the receivers are not known or if the nodes are uniformly scattered in the environment, an omni directional antenna can be employed.

Aside of the antenna gains, the polarization is the second critical aspect when this comes to the design of an energy transfer antenna design. The antennas are often designed to be vertically or horizontally polarized but in some applications, particularly the ones where the mutual position of the antennas is either not known in advance or can change (e.g., a sensor node attached a rotating structure), this may be beneficial to have a circular polarized antenna.

\section{A. Antenna Measurements}

To give a practical example how the design of antennas affects the performance of wireless energy transfer, in this section, we have characterized the amount of energy which can be harvested by the very same system using the two different antennas - a commercial dipole and the specially designed patch antenna. Both antennas are intended to operate at the frequency band centered at $868 \mathrm{MHz}$. The reason for us selecting a patch antenna was twofold. First, this type of antenna is known to feature higher directivity than the dipoles. At the very same time, these antennas are still sufficiently simple and technological for mass-scale production. Namely, the designed patch antenna for this study features the $3 \mathrm{~dB}$ beam width of 75 degrees, bandwidth of $2 \mathrm{MHz}$, and achieves the radiation efficiency of 95 percent, and the maximum gain of $6.7 \mathrm{dBi}$.

As dipole antenna we used a commercial half wave center-fed dipole antenna. The recommended frequency range by the manufacturer is from 853 to $883 \mathrm{MHz}$ and the peak gain is $-2.3 \mathrm{dBi}$.

In our experiments, we transmitted a carrier wave with the transmission power of 3530,27 and $23 \mathrm{dBm}$ feed to the transmit antenna and measured the received power after the receive antenna. Fig. 1 illustrates the results. The first four measurements are completed using the patch antennas both in transmitter and receiver, whereas in the last measurement we used the omni directional dipole antennas in both ends.

All the measurements were conducted having the distances $0.2-1.5$ meters between the transmitter and the receiver antennas. The results reveal that with the used antennas a feasible operation distance is about half a meter if the energy is intend to transmit from point to a single energy receiver. It is clearly seen that an omni directional antenna should not be used in such scenario. With patch type antennas we see that a significant amount and for many applications enough energy can be transmitted with RF signal. Note that the presented results do not account for the loss in a signal conversion to direct current (DC).

Table III summarizes the power received for the different antenna set ups depending on the distance. The second column characterizes the case when patch antennas were used in both ends, whereas the third column corresponds to the case when dipole antennas were used for transmitter and receiver. The last column shows the results for a

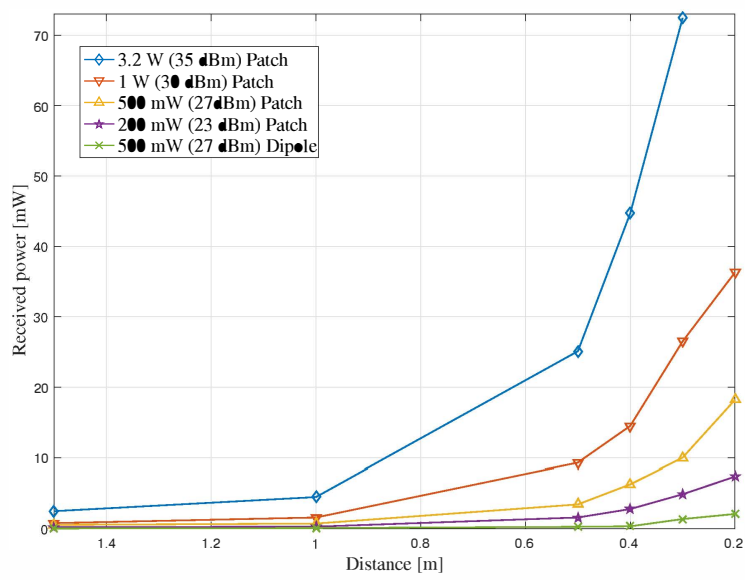

Fig. 1. Harvested energy with patch and dipole type antennas.

scenario where the energy is transmitted with a patch antenna and the receive antenna is an omni directional dipole antenna.

TABLE III. HARVESTED ENERGY WITH PATCH AND DIPOLE TYPE TRANSMIT ANTENNAS WITH 500 MW (27DBM) TRANSMISSION POWER

\begin{tabular}{cccc} 
Distance $[\mathrm{m}]$ & Patch $[\mathrm{mW}]$ & Dipole $[\mathrm{mW}]$ & Patch/Dipole $[\mathrm{mW}]$ \\
\hline 2 & 0.25 & 0.03 & 0.19 \\
1.5 & 0.55 & 0.04 & 0.22 \\
1 & 0.74 & 0.10 & 0.30 \\
0.5 & 3.46 & 0.28 & 1.17 \\
0.4 & 6.30 & 0.35 & 2.09 \\
0.3 & 10.00 & 1.38 & 3.39 \\
0.2 & 18.20 & 2.10 & 8.32 \\
\hline
\end{tabular}

\section{RF-DC CONVERTER}

Before the received energy can be utilized by a node, an RF AC signal has to be converted to DC. Even though this can be achieved in multiple ways, the diode-based rectifier circuits are nowadays typically employed for this purpose. The design of a rectifier circuitry is a non-trivial task, involving multiple decisions and compromises, some of which are discussed further.

To start with, one have to pick up the diodes to use, accounting for such characteristics as threshold and reverse-breakdown voltage, element parasitics and harmonic generation. The diode threshold and the reverse-breakdown voltage are important parameters since the voltage of an input radio signal may be quite low when it reaches the rectifying elements. Thus, if the breakdown voltage is not low enough, the incoming signal can be unable to overcome the barrier and the output capacitors will not get charged. Junction resistances and capacitances as well as the package inductances and capacitances affect the efficiency and limit the maximum operation frequency. Aside of this, the power characteristics of the diodes also need to be accounted for. Finally, the diodes nonlinearity causes frequency harmonics thus reducing the portion of energy, which is converted from RF to DC.

Next, one has to select the proper architecture of a rectifying circuitry and the number of stages to use. Few possible architecture options are discussed, e.g., in [5]. Importantly, each next rectifier stage enables to increase the sensitivity of the circuit, thus enabling it to collect energy from weaker input signals, at a cost of reducing the efficiency of energy conversion for high-power signals (as shown, e.g., in [15]). Due to this reason, the decision of the rectifier circuit to use and the number of stages must be made mindful of the expected power of the radio signal. The two other aspects which have to be accounted 
for are the costs (which inevitably increases with the increase of the stages) and the characteristics of the circuits connected after the rectifier and particularly the range of their accepted voltages.

Finally, the input of the rectifier has to be matched with the antenna in order to prevent the energy being reflected back to the environment. Impedance matching is particularly difficult for energy harvesting circuits since the impedance changes as a function of frequency and input power due to the power nonlinearity of the rectifying elements, i.e., diodes [5]. Even though in theory finding feasible LC components for matching seems to be a trivial task, we have witnessed in practice, that once all the real-life non-idealities start to play the role, the matching component selection often requires adjusting. All the discussed above results in realistic RF signal to DC peak conversion efficiency after all the losses in the energy harvesting circuit of around 50-80 percent.

For this study we have designed and produced own energy harvesting circuitry on a printed board. For the board we used FR4 with $0.8 \mathrm{~mm}$ substrate height and $0.18 \mathrm{um}$ trace thickness. The substrate dielectric is $E_{r}=4.8$. With these parameters $1.4 \mathrm{~mm}$ wide line provides $50 \mathrm{Ohms}$ impedance. We applied a two-stage Dickson charge pump built using the Avago HSMS 285C pair serial connected diodes. The selected diodes are designed and optimized for low voltage operation and feature maximum AC-DC conversion efficiency when the input signal is weak. After assembling the PCB, we measured the input impedance of the circuit with a vector network analyzer (Anritsu MS4623B). Using the measured impedance, we calculated the LC matching components for the circuit using a Smith's chart. Fine-tuning of the LC matching components was done to mitigate the imperfections and tolerances of the used components as well as the component assembly process. Any resistive components were not used in impedance matching.

The measured parameters of the designed rectifier are summarized in Table IV, which shows the effect of the power of input radio signal on the amount of collected energy from a $868 \mathrm{MHz}$ carrier wave and the efficiency of the designed charge pump. The measurement were done on a constant resistive $9 \mathrm{k}$ Ohms load.

TABLE IV. CHARGE PUMP EFFICIENCY

\begin{tabular}{cccc}
$\begin{array}{c}\text { Received power } \\
{[d B m / m W]}\end{array}$ & $\begin{array}{c}\text { Power after DC } \\
\text { conversion }[\mathrm{mW}]\end{array}$ & Max voltage [V] & $\begin{array}{c}\text { Efficiency } \\
(\%)\end{array}$ \\
\hline $5 / 10.000$ & 2.04 & 4.12 & 20 \\
$0 / 1.000$ & 0.59 & 2.21 & 59 \\
$-5 / 0.316$ & 0.16 & 1.15 & 51 \\
$-10 / 0.100$ & 0.038 & 0.56 & 38 \\
\hline
\end{tabular}

\section{NODE DESIGN}

Last but probably the most important component of the system is the actual sensor node. The supply of a node with energy harvesting dictates a whole set of specific challenges, which must be addressed in its design.

First, the possible fluctuations in the power of the input energycarrying signal results in significant fluctuations of the voltage on the output of the rectifier. To handle this, a node requires to have an efficient DC-DC converter, which will stabilize the voltage provided to the controller and the peripherals. Note that particular sensors as well as the analog to digital converters (ADC) operating with external voltage reference are very sensible to supply voltage fluctuations and may require to have a voltage within specific and rather narrow range. Albeit this is well known that the operation with higher supply voltage typically causes a device (e.g., sensor, processor or radio) to consume more energy, generation of each new supply voltage introduces the additional losses and increases the costs of a device. Due to this reason, the feasibility of enabling multiple power rails on an energy harvesting node needs to be carefully evaluated.

Second, the intermittent nature of the harvested energy income requires a node to possess an energy buffer, which can enable device to stay alive during a period when no energy income is available. The capacity of the buffer and its design are selected mindful of the application and the expected periods of energy unavailability. Typically, for this purpose are used either the supercapasitors or the secondary batteries. The former, albeit featuring low self-discharge currents and capability to be charged with low currents, often have very limited capacity and rather high costs. The latter are free of these limitations, but often need to be charged with hundreds $\mathrm{mA}$ order currents, which are hard to get with energy harvesting.

Third, the core processing system and the radio installed on an energy harvesting node need to be carefully selected. The processing core must feature low consumption both in active mode and especially in sleep mode. The radio technology should also be selected to minimize the energy consumption. In this respect the over-the-air data rate as well as the possibility of flexible transmit power control are important parameters to look at. Another important factor in this respect is the minimization of consumption for communication between the processing core and the radio. In this respect, the systemon-chip (SoC) based solution clearly outperform the standalone radio and microcontroller based systems with communication over a serial interface.

Fourth, to minimize the energy consumption, the operation of the controller should be maximally optimized and the time for it in sleep mode should be maximized. This requires careful study of the features of the controller and maximum usage of the available on it hardware features, such as direct memory access (e.g., for communication over serial interface or getting data from ADC), security and computing accelerators, etc.

Finally, the proper selection of the sensors and other peripherals is of extreme importance. As this is illustrated in Table V, not all the sensors can be easily powered with the amounts of energy which can be harvested and stored in the energy buffer of a harvesting-powered node. To give a practical example, many state-of-the art heater-based gas concentration sensors have to be preheated for hours consuming 20-50 mW before they provide an accurate measurement. Another important design metric to pay attention to is the quiescent current of a sensor. When the quiescent current consumption is high, the power line of the sensor may need to be equipped with a transistor switch, enabling the controller to power the sensor off when it is not needed.

In this study we have used a sensor node from Solmu Technologies Ltd. [16] designed and optimized specifically for energy-harvesting powered operation. A node (depicted in Fig. 2) has in its core a multi radio technology enabled SoC based on 32-bit ARM controller (consuming $100 \mathrm{nA}$ in shut down and below $3 \mathrm{~mA}$ operating with a clock of $40 \mathrm{MHz}$ ) and is equipped with a DC-DC converter enabling to convert an input voltage from $0.1 \mathrm{~V}$ to over $5 \mathrm{~V}$ to a stable predefined voltage in the range from 2 to $3.6 \mathrm{~V}$. Importantly the converter is optimized for low-power operation and shows efficiency of over $80 \%$ even having $10 \mathrm{uA}$ current on its input. As the energy buffer the sensor node uses high-volume tantalum capacitors capable of storing up to $10 \mathrm{~J}$ of energy. A coin-size lithium battery can be also used as a secondary energy source. The node is equipped with an instrumentalgrade external ADC and equipped with low-consuming environment (i.e., temperature and humidity) and 3-axis acceleration sensors .

\section{EXPERIMENTAL SETUP}

Finally, all the three components of a device discussed in the previous sections were integrated together in a single demo solution, illustrated in Fig. 3. The sensor node was attached to a rotating 
TABLE V. CONSUMPTION OF THE STATE-OF-ART COMMERCIAL SENSORS

\begin{tabular}{lll} 
Sensor type & Consumption min & Consumption max \\
\hline Movement & & \\
\hline Accleremeters & dozens $\mu \mathrm{W}$ & $\begin{array}{l}\text { hundreds } \mu \mathrm{W} \\
\text { dozens } \mathrm{mW}\end{array}$ \\
Gyroscopes & units $\mathrm{mW}$ & \\
\hline Environmental conditions & & hundreds $\mu \mathrm{W}$ \\
\hline Ambient temperature & units $\mu \mathrm{W}$ & dozens $\mu \mathrm{W}$ \\
Ambient pressure & dozens $\mu \mathrm{W}$ & hundreds $\mu \mathrm{W}$ \\
Ambient humidity & units $\mu \mathrm{W}$ & hundreds $\mathrm{mW}$ \\
Ambient light (incl.UV) & dozens $\mu \mathrm{W}$ & hundreds $\mathrm{mW}$ \\
Air quality & dozens $\mu \mathrm{W}$ & dozens $\mathrm{mW}$ \\
Gas presence & hundreds $\mu \mathrm{W}$ & units $\mathrm{mW}$ \\
Dust (optical) & dozens $\mathrm{mW}$ & \\
Smoke (optical) & hundreds $\mu \mathrm{W}$ & units $\mathrm{mW}$ \\
\hline Presence & & hundreds $\mu \mathrm{W}$ \\
\hline Magnetic/compass & hundreds $\mu \mathrm{W}$ & \\
Touch & dozens $\mu \mathrm{W}$ & dozens $\mathrm{mW}$ \\
\hline Physical conditions & & dozens $\mathrm{mW}$ \\
\hline Heart rate & units $\mathrm{mW}$ & dozens $\mathrm{mW}$ \\
Gestures (optical) & units $\mathrm{mW}$ & hundreds mW \\
\hline Other & &
\end{tabular}

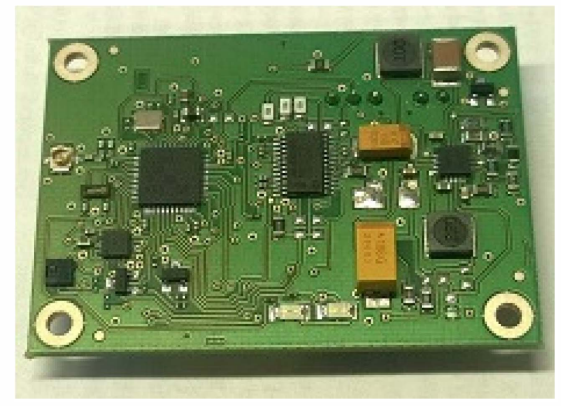

Fig. 2. An integrated sensor node by Solmu Technologies Ltd.

shift of a motor and configured to periodically measure and transmit either the temperature or the RPM (calculated from accetometers measurements). The results were transmitted to a tablet computer over a BLE link.

The energy to the system was provided by a dedicated energy transmitter which was placed at in the range of 0.5 to 1 meters from the sensor device perpendicular to the axis of shifts rotation. We have confirmed that longer distances are possible, but then the operation of a sensor node is intermittent. Meanwhile while located within 1 meter from the energy transmitter the sensor node could read sensor data, do signal processing (FFT) and transmit the data to a receiver without any batteries. Note that the antenna used in our experiments was vertically polarized and thus the receiver was not receiving the energy constantly.

The same energy harvesting circuit was used in another setup, shown in Fig. 4, integrating an LED and a printed electronic display. Since the consumption of this application is lower a less efficient omni directional antennas can be used. The LED in the application consumes approximately $12 \mathrm{~mA}$ and it gets enough power approximately from one meter distance. We transmitted energy using our inhouse made LORA radio design operating on $868 \mathrm{MHz}$ ISM band.

\section{CONCLUSIONS}

In the current paper, we focused on and reported the design of a real-life sensor node for industrial application operating with energy harvesting. First, we have analyzed the potential sources,

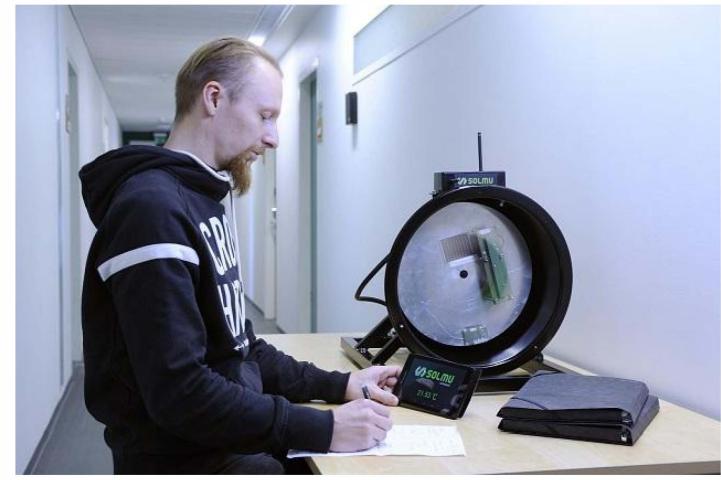

Fig. 3. Wireless sensor node application in a rotating environment enabled with energy transmission.

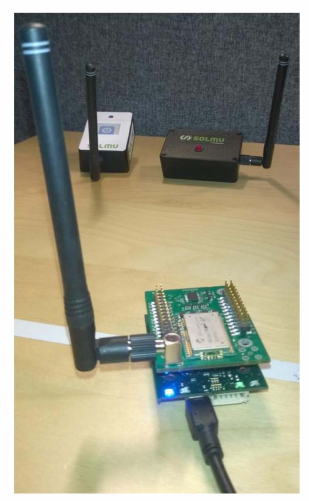

Fig. 4. Powering a printed electronic display and a conventional LED using the LORA radio and energy transmission.

from which the energy can be harvested, and concluded that the RFbased wireless energy transfer provides an attractive opportunity. The major advantage of it is the possibility to enable a more stable and controllable income of the energy compared to more opportunistic energy harvesting from light or vibration. Second, we have discussed the frequency regulations, showing that even under them almost uninterrupted energy transfer can be enabled. Third, we have discussed the design aspects for the three major components of a real-life wireless energy transfer powered sensor nodes, namely its energy receiving antenna, rectifying circuit and the sensor node itself. Importantly, all the discussions are backed up by the practical implementation and evaluation activities. Finally, all the three components of the system have been integrated together and the resulting system was tested in a challenging rotating environment as a part of a demo application. These results have confirmed the feasibility of our approach and demonstrated the potential possibility of developing the industrial sensory applications powered solely with the energy transferred over an RF channel. In future, we plan to use the developed test bed for evaluating and comparing the various design solutions and approaches as well as for prototyping the novel applications and use cases. In this respect the developed system is seen as a potential component of the $5 \mathrm{G}$ and IoT activities of the University of Oulu conducted within the $5 \mathrm{G}$ test network $(5 \mathrm{GTN})$ initiative.

\section{ACKNOWLEDGEMENTS}

This research was financially supported by the Academy of Finland. The devices in experimental setups were offered by Solmu Technolo- 
gies Ltd. and antennas were designed by CWC Nippon, University of Oulu Research Institute in Japan.

\section{REFERENCES}

[1] J. Hill, System architecture for wireless sensor networks. University of California: Berkeley, 2003.

[2] M. Paavola and K. Leivisk, Wireless sensor networks in industrial automation. INTECH Open Access Publisher, 2010.

[3] IEEE, "IEEE Standard 802.15.4: Wireless medium access contro (MAC) and physical layer (PHY) specifications for low-rate wireless personal area networks," IEEE, Tech. Rep., 2010.

[4] I. Popa, J. Janhunen, and K. Mikhaylov, "Performance evaluation of zigbee and uwb wireless sensors under doppler effect in rotating mechanical structures," in European Wireless 2016; 22th European Wireless Conference, May 2016, pp. 1-6.

[5] C. R. Valenta and G. D. Durgin, "Harvesting wireless power: Survey of energy-harvester conversion efficiency in far-field, wireless power transfer systems," IEEE Microwave Magazine, vol. 15, no. 4, pp. 108120, June 2014.

[6] A. Nimo, D. Grgic, and L. M. Reindl, "Optimization of passive low power wireless electromagnetic energy harvesters," Sensors, vol. 12 , no. 10, p. 13636, 2012. [Online]. Available: http://www.mdpi.com/1424$8220 / 12 / 10 / 13636$

[7] NREL, "Best research-cell efficiencies," National Renewable Energy Laboratory, Tech. Rep., 19.2.2017, [Online]. available: https://www.nrel.gov/pv/assets/images/efficiency-chart.png.

[8] M.-G. Kang, W.-S. Jung, C.-Y. Kang, and S.-J. Yoon, "Recent progress on pzt based piezoelectric energy harvesting technologies," Actuators, vol. 5, no. 1, 2016. [Online]. Available: http://www.mdpi.com/2076$0825 / 5 / 1 / 5$

[9] E. Sordiashie, Electromagnetic Harvesting to Power Energy Management Sensors in the Built Environment. University of NebraskaLincoln, 2012.

[10] D.-X. Yang, Z. Hu, H. Zhao, H.-F. Hu, Y.-Z. Sun, and B.-J. Hou, "Through-metal-wall power delivery and data transmission for enclosed sensors: A review," Sensors, vol. 15, no. 12, pp. 31 581-31 605, 2015. [Online]. Available: http://www.mdpi.com/1424-8220/15/12/29870

[11] T. Nylnden, J. Boutellier, K. Nikunen, J. Hannuksela, and O. Silvn, "Low-power reconfigurable miniature sensor nodes for condition monitoring," International Journal of Parallel Programming, pp. 1-21, 2014. [Online]. Available: http://dx.doi.org/10.1007/s10766-013-0302-5

[12] Figora, "Mrys luvasta vapaiden radiolhettimien yhteistaajuuksista ja kytst," Figora, Finnish Communications Regulatory Authority, Tech. Rep., 2016.

[13] ETSI, "ETSI EN 300 200-1,electromagnetic compatibility and radio spectrum matters (erm); short range devices (srd); radio equipment to be used in the $25 \mathrm{mhz}$ to $1000 \mathrm{mhz}$ frequency range with power levels ranging up to $500 \mathrm{mw}$; part 1: Technical characteristics and test methods," ETSI, Tech. Rep., 2012.

[14] W. Brown, "The history of power transmission by radio waves," IEEE Transaction on Microwave Theory and Technoques, vol. 32, no. 9, pp. 1230-1242, Sep. 1984.

[15] M. H. Ouda, W. Khalil, and K. N. Salama, "Wide-range adaptive rfto-dc power converter for uhf rfids," IEEE Microwave and Wireless Components Letters, vol. 26, no. 8, pp. 634-636, Aug 2016.

[16] Solmu Technologies Ltd., Tech. Rep., 19.2.2017, [Online]. available: http://www.solmutech.com/. 